\title{
A CONSERVATION MODEL FOR BLACK RHINO
}

\author{
John W. Hearne, Johan Swart and Peter Goodman ${ }^{\dagger}$ \\ Department of Mathematics \& Applied Mathematics \\ University of Natal \\ Pietermaritzburg 3200 \\ South Africa
}

† Natal Parks Board, Mkuzi Game Reserve, Mkuze 3965, South Africa

\begin{abstract}
Over the past thirty years the black rhinoceros (Diceros bicornis) population in Africa has declined from about 65000 to 3500 . In contrast the South African and Namibian population has increased four-fold to 1000 over the same period. The recently developed national conservation strategy for black rhino has as its main goal a further four-fold increase in the current population in as short a period as possible. To achieve this, the growth rate of the population as a whole will have to be maximised. This involves removing animals from areas where the population is approaching the ecological carrying capacity and establishing new viable populations in other suitable reserves.

A model, incorporating what is known about the population biology of black rhino, was developed to give guidance to managers on the most appropriate harvesting strategy to adopt for their populations; in particular, to determine the rate of removals and the age and sex of individuals to be removed to attain the conservation goal as scon.as possible.
\end{abstract}

\section{Introduction}

Over the past decade, black rhinoceros (Diceros bicornis) have continued to decline in Africa (Cumming,1987). A policy was recently developed for this species in Southern Africa (Brookes,1989) which, it is hoped, will enhance the survival prospects of the black rhino. One of the primary aims stated in this policy is to increase the current population of approximately 600 rhino of the southern-central subspecies (Diceros bicornis minor), found mainly in Natal and the Kruger National Park, to at least 2000 in as short a period as possible. To achieve this, the growth rate of the subspecies as a whole will have to be maximised. This involves removing animals from areas where the population is approaching the ecological carrying capacity and establishing new viable populations in other suitable reserves.

The population and behavioural biology of black rhinoceros is not well understood. Our current understanding stems from the several different sub-species of black rhino which occur in various localities throughout east and southern Africa. The model we develop, incorporates 
information on black rhino population chatacteristics from the published literature and other unpublished sources. Where no specific data for black rhino exists, we substitute with empirically supported generalisations for large mammals from the literature notably Eberhardt (1977),Fowler (1981) and Laws (1981).

The model was developed to give guidance to managers on the most appropriate harvesting strategy to adopt for their populations; in particular, to determine the rate of removals and the age and sex of individuals to be removed to attain a 2000 strong Southern African population as soon as possible.

\section{Formulation of the Model}

Black rhinos available for translocation are found in the following game reserves: Hluhluwe Umfolozi, Mkuzi, Ndumu and Itala [Brooks 1989]. To keep the model simple, the populations from these reserves will be regarded as constituting a single group, referred to as the Founder Population. In the sarne way translocated black rhinos will be regarded as a single group referred to as the Translocated Population.

In order to preserve genetic diversity the Founder Population will be kept close to the estimated ecological carrying capacity and a removal policy will only be regarded as feasible if the adult female and male population numbers do not decline to less than $60 \%$ of their initial values at any time. Genetic diversity will be further managed when selecting animals for translocation and in selecting their destination.

The Founder population is divided into 8 groups according to age and sex. The various female groups, their initial values and the rates determining their levels are shown in table 1. The male groups M1 - M4 are divided in a similar way.

\begin{tabular}{|l|c|c|c|c|}
\hline Group & $\begin{array}{c}\text { Initial } \\
\text { Value }\end{array}$ & $\begin{array}{l}\text { Age } \\
(\mathrm{yrg})\end{array}$ & Flows IN & Flows OUT \\
\hline$F_{1}$ & 10 & $0-1$ & Births & Maturation, death, predation \\
$F_{2}$ & 8 & $1-2$ & Maturation & Maturation, death, predation, \\
$F_{3}$ & 22 & $2-8$ & Maturation & Maturation, death, removal \\
$F_{1}$ & 160 & $8+$ & Maturation & Death, removal \\
\hline
\end{tabular}


From the table it should be noted that migration does not take place and that predation is confined to the younger groups. Calves stay with the cows until at least age 2 and removal (translocation) is restricted to the groups $F_{3}$ and $F_{4}$ (as well as $M_{3}, M_{4}$ ). In this article the symbols $F_{i}$ and $M_{i}(i=1, \cdots, 4)$ will also be used to denote the numbers in the corresponding groups.

A juvenile black rhino exerts less pressure on resources than an adult and we therefore define the adult equivalent population (AEP) as

$$
A E P=a_{1}\left(F_{1}+M_{1}\right)+a_{2}\left(F_{2}+M_{2}\right)+a_{3}\left(F_{3}+M_{3}\right)+F_{4}+M_{4}
$$

where $0 \leq a_{i} \leq 1(i=1,2,3)$.

Population density is measured as the ratio $\frac{A E P}{\text { ECC }}$ where ECC is the estimated ecological carrying capacity in adult units.

Observations indicate that births in Umfolozi Game Reserve have occurred as early as 6 years (Goddard 1967) and in the high density extreme in Hluhluwe Game Reserve first calves are dropped at around 10 years and 6 months. (Hitchins and Anderson 1983). In this model it is assumed that birthe occur only amongst the group $F_{4}$ (which is true on average).

Fecundity, which is reflected by the interval between calving is suggested to be a declining function of density (Eberhardt 1977, Laws 1981). Ilitchins and Anderson (1983) summarised calving interval statistics from a variety of areas and recorded a minimum between calving of 26 months (0.46 calves/year/cow) and a maximum of 63 months ( 0.19 calves/year/cow). The exact shape of the fecundity function FF is not known for rhino and we have assumed a smooth decreasing sigmoidal function of density between the recorded maximum and minimum values of 0.46 and 0.19 respectively.

The effect of density changes on the conception rate is represented by $F F_{1}$, a first-order delayed version of the fecundity function $F F$, with delay time $T_{1}$. Thus

$$
\frac{d}{d t} F_{1}=\left(F F-F F_{1}\right) / T_{1}
$$

It is only after some further delay (gestation period) that density-induced changes in the conception rate result in changes in the birth rate. Thus $F_{1}$ is subjected to a further delay to yield the specific birth rate $\mathrm{FF}_{4}$. This delay is madelled by the third-order delay equations

$$
\frac{d}{d t} F F_{i}=\left(F F_{i-1}-F F_{i}\right) / T_{2}, \quad i=2,3,4
$$


where $\mathrm{FF}_{2}$ and $\mathrm{FF}_{3}$ are intermediate variables and $3 \mathrm{~T}_{2}$ is the gestation period. The birth rate is therefore given by

$$
\text { birth rate }=F_{4} \cdot \mathrm{FF}_{4} \quad(\text { calves } / \mathrm{yr})
$$

Mortality is a function of age (Goddard 1970) and density (Eberhardt 1977, Fowler 1981). Eberhardt (1977) proposed that one of the first signs of density dependent stress was an increase in juvenile mortality. At the other extreme Fowler (1981) states that in many large mammal populations, adult survival is insensitive to changes in density. Observations indicate that for rhino, subadults have higher mortality rates than adults because they are subject to more social stress than adults, particularly when they are trying to establish their home ranges for the first time. Animals in the two youngest age groups are also subject to nutritional stress. Yearlings (age group 2) are weaned and so must depend on vegetation for food. They therefore have a higher mortality rate and are more susceptible to density stress than unweaned calves. There is insufficient information available for a precise definition of the mortality functions but based on the above discussion and field experience the functions shown in figure 1 were considered plausible. In addition to natural mortality, deaths due to predation occur amongst the two younger groups.
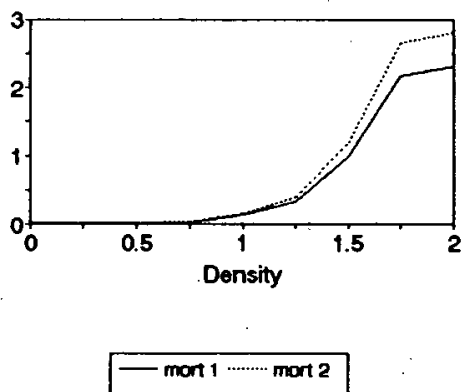

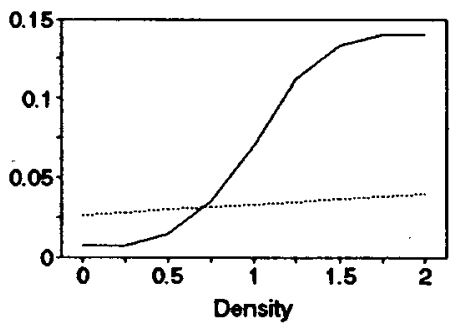

mort 3 ......... mort 4

Fig. 1

The mortality functions for the different groups

The population is assumed to be uniformly distributed over each age group and ageing is assumed proportional to the number of animals in each group (excluding the adult groups). 
Observations indicate that half of all rhino births are female. The female sector of the Founder Population is therefore described by the following model equations:

$$
\begin{aligned}
& \frac{d}{d t} F_{1}=0.5\left(F_{4} \cdot F F_{4}\right)-F_{1} \cdot F_{1} A N-F_{1} \cdot \operatorname{mort}_{1}-F_{1} \cdot F_{1} P N \\
& \frac{d}{d t} F_{2}=F_{1} \cdot F_{1} A N-F_{2} \cdot F_{2} A N-F_{2} \cdot \operatorname{mort}_{2}-F_{2} \cdot F_{2} P N \\
& \frac{d}{d t} F_{3}=F_{2} \cdot F_{2} A N-F_{3} \cdot F_{3} A N-F_{3} \cdot \operatorname{mort}_{3}-f_{3} \\
& \frac{d}{d t} F_{4}=F_{3} \cdot F_{3} A N-F_{3} \cdot \text { mort }_{4}-F_{4}
\end{aligned}
$$

where $\quad F_{i} A N=$ ageing normal of group $F_{i}\left(y^{-1}\right)$

$$
\begin{aligned}
& \text { mort }_{\mathbf{i}}=\text { mortality function of group } F_{i}\left(\mathrm{yr}^{-1}\right) \\
& F_{i} P N=\text { predation normal of group } F_{i}\left(y^{-1}\right)
\end{aligned}
$$

The removal rates $f_{3}$ and $f_{1}$ for groups 3 and 4 respectively are exogenously specified. The mortality functions are given in fig. 1 , the ageing normals $F_{i}$ AN $(i=1,2,3)$ are $1,1, \frac{1}{6}$ and the predation normals $F_{i}$ PN $(i=1,2)$ are 0.16 and 0.01 .

The male sector is modelled in a similar way as is the translocated population. The removals from the founder groups $F_{3}, F_{1}, M_{3}$ and $M_{4}$ are additional flows into the translocated population.

Until now removals have been conservative and aimed only at dropping the founder population level below the estimated ecological carrying capacity in order to stimulate breeding and survival. Initial values for both female and male translocated rhinos are $0,0,5,20$ for the four age groups respectively.

The above model comprises a system of nonlinear first order differential equations. A Runge-Kutta-Fehlberg algorithm was used to solve the system numerically for various translocaton strategies. The aim was to identify strategies which maximise the total (founder + translocated) rhino population over a 30 year period.

\section{Resulls}

For this set of results constant removals take place once per year. A policy is considered feasible if after removal at any time during the 30 year simulation $F_{3} \geq 1, M_{3} \geq 1, \quad F_{4} \geq 100$, 
$M_{4} \geq 100$. The adult founder groups need to be kept reasonably high to preserve genetic diversity. The vector $\left(\begin{array}{lllll}f_{3} & f_{4} & m_{3} & m_{4}\end{array}\right)$ describes a removal strategy where once a year $f_{i}$ animals are removed from founder group $F_{i}$ and $m_{i}$ animals are removed from founder group $M_{i}$ $(i=3,4)$. It is assumed that the ecological carrying capacity (in adult units) of the translocated population is 1600 as opposed to the 400 of the founder population.

In table 2 a comparison of some feasible removal strategies is shown. For low removals $(2-8)$, best results (ie. highest total population) are obtained by relatively high $F_{3}$ and $F_{4}$ removals. For medium removals $(10-16)$, best results are obtained by relatively high $F_{4}$ and $M_{4}$ removals and for high removals $(18-26)$, relatively high $F_{3}$ and $M_{3}$ removals are indicated. In the case of 24 and 26 removals there are few feasible strategies and it becomes meaningless to differentiate between good and poor strategies.

Figure 2 shows the total population numbers after 30 years corresponding to the best removal strategy for each constant total annual removal, while figure 3 shows the minimum number of years to reach a target population of 2000 as a function of the number of removals per year. In Figure 4 the population dynamics of the total black rhino population is compared under a removal policy of 16 animals per annum and a policy that abandons further removals. Figure 5 shows the population structure of the female sector of the founder population under the removal strategy ( $\left(\begin{array}{llll}2 & 6 & 0 & 8\end{array}\right)$. 


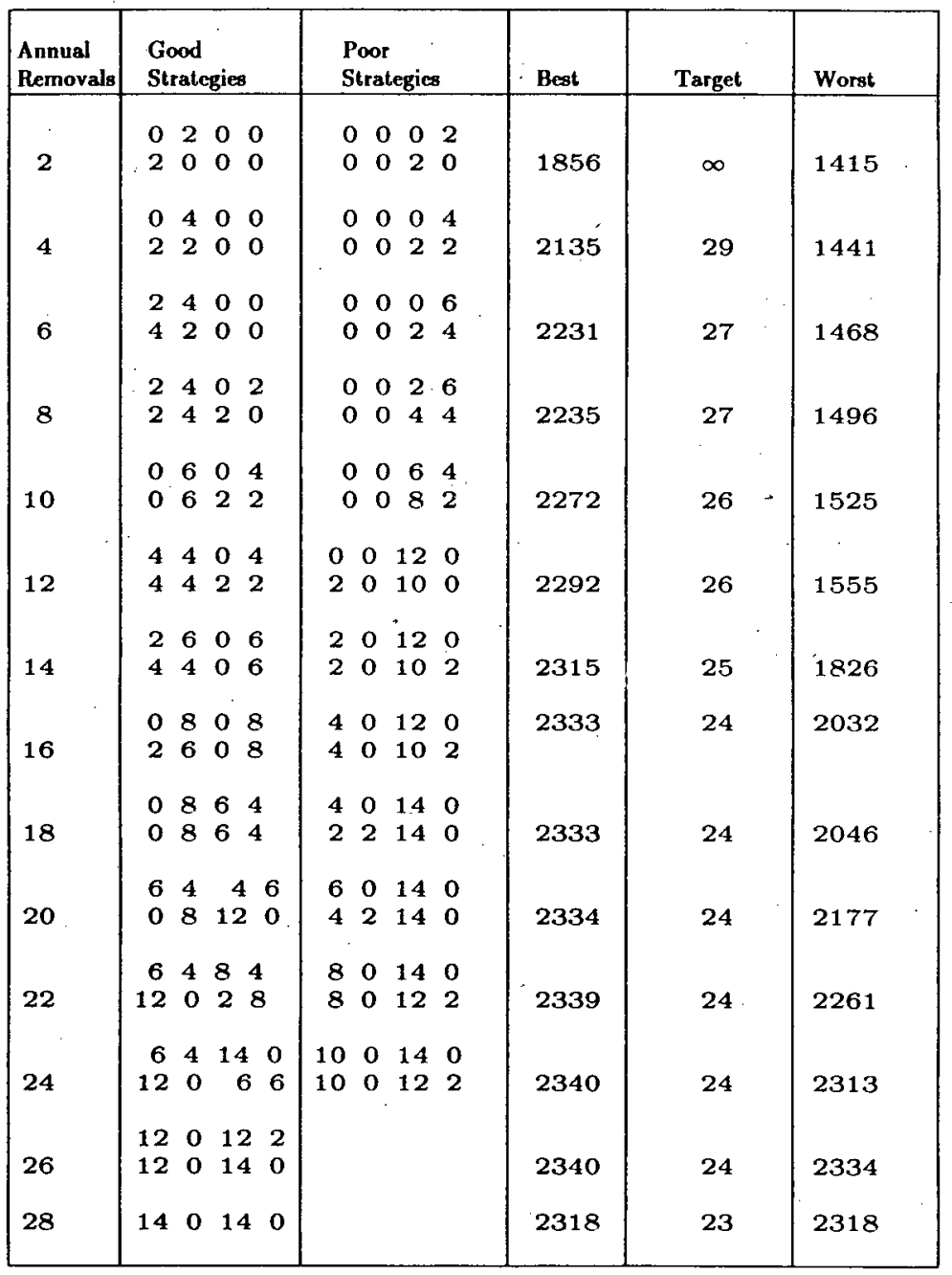

Table 2

Analysis of translocation strategies

Column 1 shows the total number of animals translocated, columns 2 and 3 list some good and poor strategies respectively. Column 4 gives the total populations after 30 years corresponding to the best strategy and column 5 indicates the first time the population exceeds 2000 . The last column shows the population after 30 years corresponding to the worst strategy. 


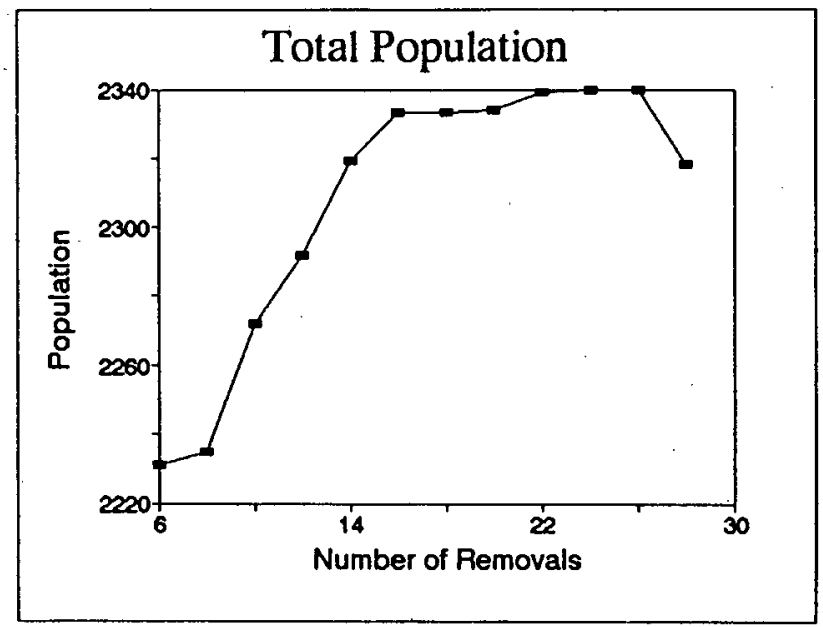

Fig. 2

Total population after 30 years corresponding to the best removal strategy for each constant total annual removal.

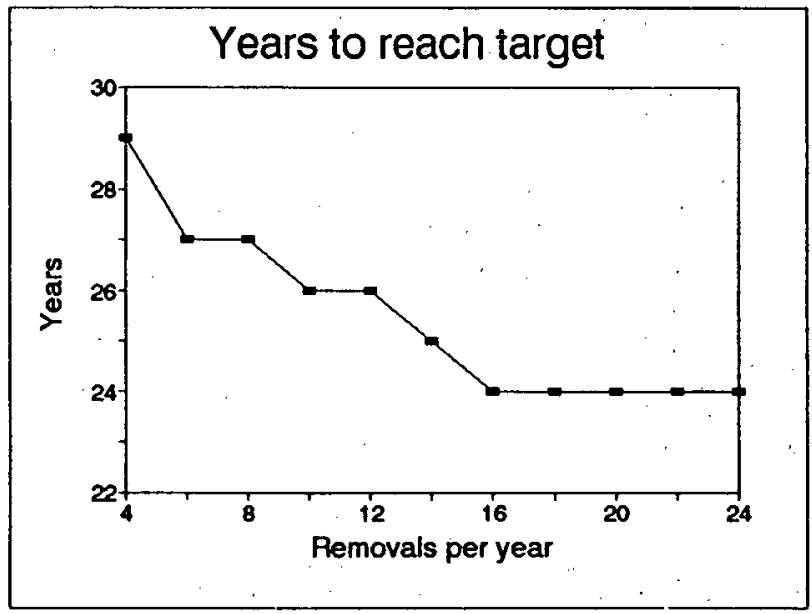

Fig. 3

Minimum number of years to reach a target population of 2000 plotted against the number of removals per year. 


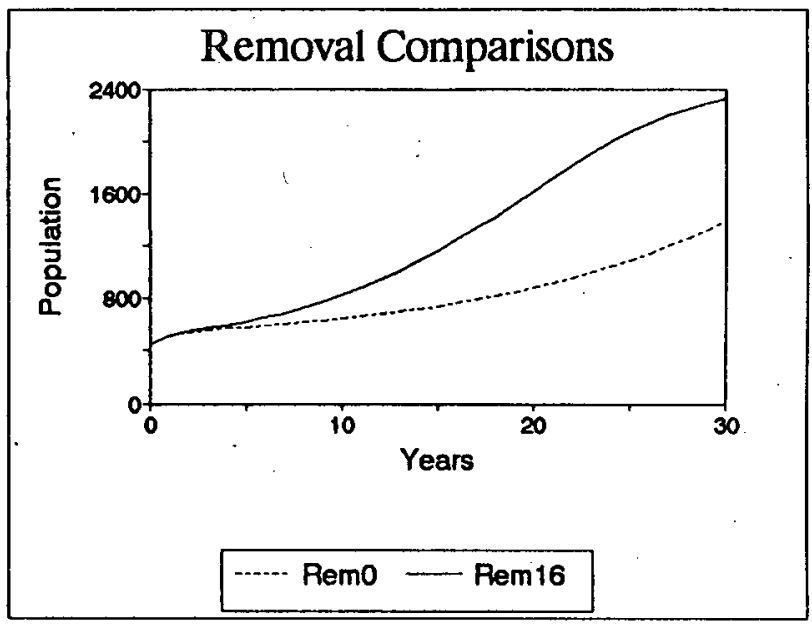

Fig. 4

The population dynamics of the total black rhino population is compared under two removal strategies. The dotted graph shows the population dynamics if no further removals were to take place, whereas the graph Rem 16 shows the population dynamics if the constant removal strategy $\left(\begin{array}{lll}2 & 60 & 8\end{array}\right)$ is applied.

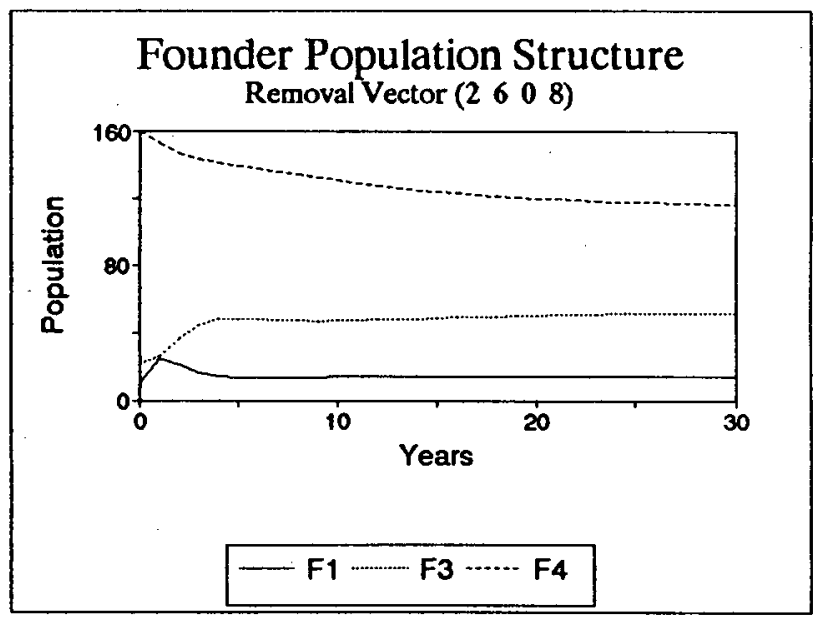

Fig. 5

The population structure of the female sector of the founder population under the removal strategy ( $\left.\begin{array}{llll}2 & 6 & 0 & 8\end{array}\right)$. The graph for $F_{2}$ is almost identical to that for $F_{1}$ and is not shown. 


\section{Sensitivity Analysis}

\section{Initial Values}

The above results are only valid for the particular set of initial values used. To obtain some indication of the effect of the initial values some further simulations were performed. The initial values of the oldest male and female groups were both reduced by 15 animals, a reduction of nearly $10 \%$, and these animals were then distributed equally amongst the younger groups. With these initial values it was found that 'good' strategies remained good strategies and that generally most strategies were surprisingly robust to changes in the initial values. However, the strategy ( $\begin{array}{lll}6 & 4 & 8\end{array}$ - 4) became infeasible in the sense described earlier, thus indicating a need for caution. New changed census data or sudden changes in population structure, from poaching for example, would necessitate a repeat of the simulations.

\section{Parameters and Functions}

All the results presented so far have been based on the assumption that there is no mortality associated with the capture and translocation of rhino. In practice some removal deaths are likely to occur and in figure 6 the effect of an extreme $10 \%$ removal death rate is -illustrated. As expected the total population is lower, but as before there is little to gain from increasing the annual removal rate beyond 16 .

Mortality and fecundity are functions of density and hence depend on the specified ecological carrying capacities. The carrying capacity of the founder population is fixed but that of the translocated population depends on the amount of suitable habitat made available. In figure 7 the number of years to reach the target population of 2000 is compared under ECC specifications of 1600 and 2400 respectively for the translocated population. The target is reached 2 years earlier and once again a removal rate of 16 animals per year is the lowest removal rate to achieve this result. 


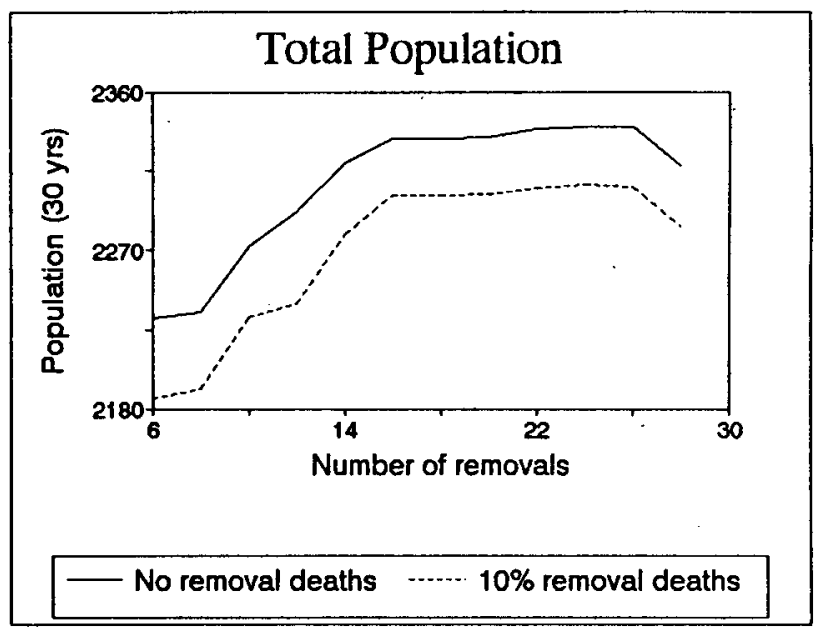

Fig. 6

The effect of translocation deaths.

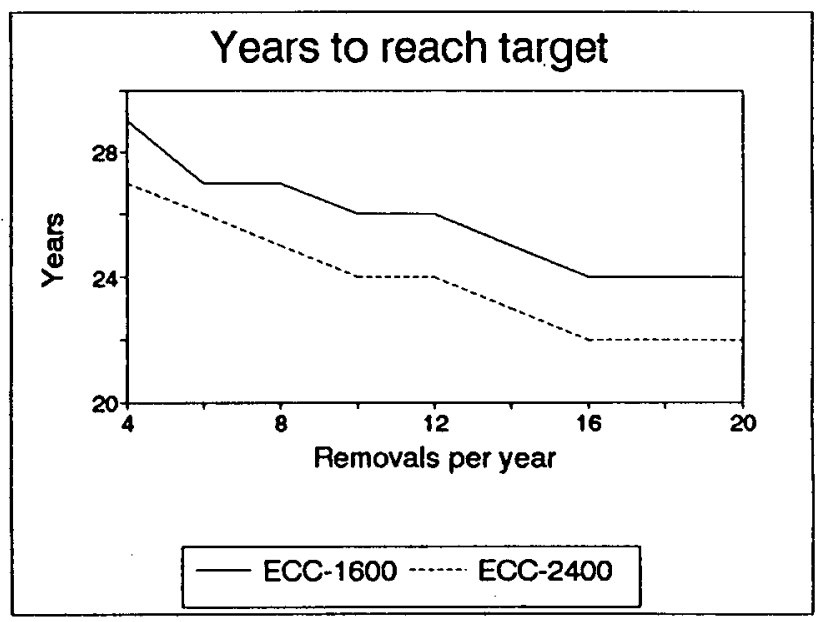

Fig. 7

The effect of carrying capacity on the time to reach the target population. 


\section{Conciusion}

The model confirms that a policy of translocation is essential if the total population of black thino modelled is to increase from 400 to a genetically viable population of well over 2000 in 25 years.

No appreciably better total population results are obtained by increasing the annual removal rate from 16 to 26 (after which a constant removal strategy is no longer feasible).

As expected the translocated population will benefit from more suitable habitat being made available. The population numbers will be higher and the target population will be reached sooner. Only a slight improvement in overall numbers results from increasing the annual removal rate from 16 to 28 and in view of the additional disturbance to the rhino as well as additional costs, a constant removal strategy of 16 is indicated, with the animals selected from appropriate groups to maximise the total population.

\section{Acknowledgement}

This project was funded by the University of Natal Research Fund, the Foundation for Research Development and the Natal Parks Board.

\section{References}

Brooks, P. M. 1989. Conservation plan for the black rhinoceros Diceros bicornis in South Africa, the TVBC States and SWA/Namibia. Unpublished report, Natal Parks Board, Pietermaritzburg.

Cumming, D. 1987. Zimbabwe and the conservation of black rhino. Zimbabwe Science News 21: $59-62$.

Eberhardt, L. L. 1977. Optimal policies for conservation of large mammals, with special reference to marine ecosystems. Environmental Conservation 4: 205-212.

Fowler, C. W. 1981. Comparative population dynamics in large mammals. In: C. W. Fowler, and T. D. Smith (eds) Dynamics of Large Mammal Populations. J. Wiley \& Sons, New York.

Goddard, J. 1967. Home range, bchaviour, and recruitment rates of two black rhinoceros populations. East African Wildlife Journal 5: 133-150. 
Goddard, J. 1970. Age criteria and vital statistics of a black rhinoceros population, East African Wildife Journal 8: 105-122.

Hitchins, P. M. 1978. Age determination of the black rhinoceros (Diceros bicornis Linn) in Zululand, South African Wildlife Journal Res. 8: 71-80.

Hitchins, P. M. and Anderson, J. L. 1983. Reproduction, population characteristics and management of the black rhinoceros Diceros bicornis minor in the Hluhluwe/Corridor/Umfolozi Game Reserve Complex. South African Wildife Journal Res. 8: 71-80.

Laws, R. M. 1981. Experiences in the study of large marnmals. In : C. W. Fowler and T. D. Smith (eds) Dynamics of Large Mammal Populations. J. Wiley \& Sons, New York. 